\title{
Mini-symposium
}

\section{Infective endocarditis: introduction}

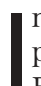
nfective endocarditis is a very complex disease with a serious prognosis. Even if it is not a very common disease most cardiologists are faced with a few number of cases each year, and every case is a difficult one. In the recent Euro Heart Survey for valvar diseases, 5001 patients with valvar heart disease were included during a three month period from 27 different European countries. Three per cent of them were patients with infective endocarditis and this figure was similar in the different European regions analysed. In recent years advances in echocardiography have resulted in better and earlier diagnosis, and surgical techniques and indications have also changed. In the most recent series including the French registry $^{2}$ and the Euro Heart Survey data, ${ }^{1}$ surgery was used in the active phase of the disease in more than 50\% of patients.

Even if improvements in both the diagnosis and treatment have occurred mortality figures have not dramatically changed. This lack of improvement in prognosis might be due to the fact that endocarditis is now occurring in old people, in patients unaware of having a cardiac valve disease, in patients with prosthetic valves, and is being caused by aggressive organisms such as staphylococci. Therefore every effort is needed in the field of diagnosis and management strategies. The clinical suspicion of endocarditis should be increased, not only within the scope of patients with previously known heart disease. Clinicians should think of infective endocarditis in every patient presenting with fever and vascular phenomena, even in patients with previously unknown heart disease which represents more than $50 \%$ of endocarditis cases. Once the diagnosis is suspected early diagnosis and treatment are mandatory, therefore early access to microbiological and echocardiographic studies are needed. Once the diagnosis is established adequate antibiotics should be used and decisions regarding surgical treatment should be made early and surgery should be performed, when needed, without unnecessary delays.

In his paper Dr Prendergast describes the new diagnostic criteria and explains the difficulties in the diagnosis of the disease, especially in cases with negative blood cultures stressing the need to identify the pathogen using new techniques. Dr Evangelista clarifies the role of echocardiography in the diagnosis of the disease and in the search and diagnosis of complications, and provides a comprehensive scheme for the use of transthoracic and transoesophageal echocardiography. Finally, Dr Delahaye presents a comprehensive practical discussion on surgical indications and timing of surgery. His paper reflects both his large personal experience and the review of the literature and guidelines on this difficult topic.

P Tornos
Servicio de Cardiología, Hospital Vall
d' Hebron, Barcelona Spain;
ptornos@vhebron.net

\section{REFERENCES}

1 lung B, Baron $G$, Butchart $E$, et al. A prospective survey of patients with valvular heart disease in Europe: the Euro Heart Survey on valvular heart disease. Eur Heart J 2003;24:1231-43.

2 Hoen B, Alla F, Selton-Suty CH, et al. Changing profile of infective endocarditis. Results of a 1 year survey in France. JAMA 2002;288:75-81.

\section{ELECTRONIC PAGES}

\section{Heart Online case reports: www.heartjnl.com}

T follow electronic only articles are published in conjunction with this issue of Heart.

\section{Failure of intravascular ultrasound to identify the site of recurrent focal coronary spasm during stenting}

T Azeem, D Adlam, A Gershlick

Focal coronary spasm is often associated with an area of mural plaque disease. This report describes a patient with recurrent severe coronary spasm unresponsive to medical treatment. Coronary arteriography and intravascular ultrasound identified a candidate area of minor coronary atheromatous disease but ergonovine provocation testing showed the spastic coronary segment to be distal to and distinct from this area. Coronary stenting of the site identified by ergonovine provocation testing was effective in relieving provoked and spontaneous spasm.

(Heart 2004;90:e33) www.heartjnl.com/cgi/content/full/90/ 6/e33
Candida endocarditis with mycotic pulmonary emboli following re-do Rastelli operation N T Wijesekera, M N Sheppard, M J Mullen

A case of a 19 year old patient with Candida endocarditis complicated by pulmonary infarction and pulmonary mycotic abscesses following replacement of a right ventricle to pulmonary artery homograft conduit is presented. Despite preceding hospital admissions with probable septic pulmonary emboli, diagnosis was made only after massive pulmonary haemorrhage that ultimately proved fatal. This case highlights that Candida endocarditis should be considered in patients with symptoms and signs compatible with bacterial endocarditis when blood cultures are negative, especially in the setting of congenital cardiac malformations, and illustrates the high mortality associated with delayed diagnosis.

(Heart 2004;90:e34) www.heartjnl.com/cgi/content/full/90/ $6 / \mathrm{e} 34$ 